\title{
On barring the vascular gateway against severe COVID-19 disease
}

\section{George Perdrizet ${ }^{1} \cdot$ Lawrence E. Hightower ${ }^{2}$}

Published online: 26 May 2020

(C) Cell Stress Society International 2020

Medical understanding of COVID-19 disease grows daily (Guan et al. 2020; Huang et al. 2020). Infection by SARSCoV-2 most commonly results in a self-limited viral syndrome characterized by flu-like symptoms lasting 1-2 weeks. More aggressive disease manifestations are seen in the elderly and in younger individuals with chronic diseases known to compromise host resistance to infection (Grasselli et al. 2020). Presently there are no pharmaceutical treatments known to be safe and effective for treating COVID-19 disease. The overall mortality rate of COVID-19 disease is estimated to be $1 \%$. Once hospitalized, there is a $15-62 \%$ risk of death despite the administration of emergency and critical care therapies, including mechanical ventilation, intravenous fluids, vasopressor therapy, and renal replacement therapy (Murthy et al. 2020).

The pathophysiology and clinical syndrome related to SARS-CoV-2 infection are becoming known including the systemic nature of this disease (Li et al. 2020; McGonagle et al. 2020). While pulmonary injury is the early and dominant clinical feature of active infection, there are widespread systemic effects and manifestations. Many clinical features are similar to the general pattern seen during systemic infection by other microbial organisms and run the gamut in severity from mild fever (systemic inflammatory response syndrome, SIRS) to the more severe sepsis and septic shock syndromes (Li et al. 2020). If patients survive these stages, they often acquire a secondary bacterial or fungal infection, repeating the cycle of SIRS-sepsis-shock, that becomes lethal. Alternatively, others may develop a common but poorly understood syndrome in which individual organ systems begin

Lawrence E. Hightower

Lawrence.hightower@uconn.edu

1 Advanced Wound Care and Hyperbaric Medicine, The Hospital of Central Connecticut, 5 Highland Street, New Britain, CT 06052, USA

2 Department of Molecular and Cell Biology, University of Connecticut, Storrs, CT 06269, USA to fail, so called multiple organ dysfunction syndrome, which is often lethal as well.

There are several features of severe systemic infections and overwhelming immune responses seen in COVID-19 which we suspect represent potential therapeutic targets for systemic therapies (Fu et al. 2020). SARS-CoV-2 results in widespread injury to the endothelial lining of blood vessels (Varga et al. 2020). This same group found evidence of viral infection of endothelial cells of glomerular capillary loops of a patient as well as diffuse endothelial inflammation, yet another pathway to organ damage. SARS-CoV-2 binds to angiotensinconverting enzyme 2 receptors that are present in the lung, heart, kidney, and intestine in addition to vascular endothelial cells. Vascular injury in general leads to loss of the integrity of the vascular system and results in two complicating phenomena - intravascular thrombosis (macro and micro) and capillary leak (Magro et al. 2020). Damaged endothelium leads to exposure of powerful procoagulants of the vessel wall, blood clots form, and blood flow stops. Major vessel thrombosis is associated with end organ infarctions - stroke, myocardial infarction, and renal failure (Gopalakrishnan et al. 2020). Microvascular thrombosis impairs perfusion and oxygen delivery to all tissues. Thus, tissue level hypoxia ensues which itself is pro-inflammatory, establishing a vicious cycle of inflammatory hypoxia. Capillary leak results in the extravasation of intravascular cells, proteins, and fluid into all tissues and is manifested clinically by tissue edema, such as pulmonary edema, cerebral edema, and soft tissue/dermal edema. This edema fluid expands the extracellular space, further worsening inflammatory hypoxia. These are systemic processes happening independent of organ or tissue type. More recently, a rare pediatric syndrome associated with COVID-19 disease, and similar to Kawasaki Disease, has been described and is characterized by systemic inflammation and organ dysfunction (Han and Lee 2018; Nathan et al. 2020). The vascular injury described in Kawasaki disease is associated with coronary artery aneurysms and is direct evidence of vascular injury (Marrani et al. 2018).

Currently drug therapies are targeted at either impairing SARS-CoV-2 viral replication (remdesivir), transferring 
immunity passively (convalescent serum, monoclonal antibodies), or suppressing the exaggerated immune response using hydroxychloroquine and anti-Il6 cytokine therapies (Sciascia et al. 2020). None of these treatments is designed to enhance the tissue level protection from direct injury by the virus nor from the indirect injury associated with inflammatory hypoxia. We have been interested in a novel therapeutic approach that is systemic and cytoprotective for conditioning tissues against planned stressful events such as major cardiovascular surgery and organ transplantation (Perdrizet 1996). At that time, it was known that cells respond to viral infection (Collins and Hightower 1982), hyperthermia (Ritossa 1962), cerebral ischemia (Currie and White 1981), and mechanical tissue injury (White 1981) by the increased synthesis of a class of proteins known as heat shock proteins, now known as members of the molecular chaperone class of proteins. The synthesis of these proteins is transiently associated with a powerful, protective cellular response that can enhance survival of cells, tissues, and whole organisms during exposure to potentially lethal events (Solomon et al. 1991). In contrast, there is evidence in support of the hypothesis that some viral infections may raise a deficient or impaired cell stress response making tissues vulnerable to damage (Hooper et al. 2012).

Whole-body hyperthermia or heat shock was used as a systemic stressor to induce protection of renal transplant organs during cold storage, likely through preservation of the microcirculation (Perdrizet et al. 1989). Acute reperfusion of ischemic organs is always followed by acute inflammation, capillary leak, and microvascular thrombosis, the same mechanisms described in severe COVID-19 disease. A major mechanism of the observed protection is thought to be related to the stress-induced synthesis of the 70-kDa heat shock protein-HSP70, now known as human HSPA1A and subsequent protection of organ blood vessels. Importantly, this same HSP70 protein had been found in stressed brain tissues where it co-located with the microvasculature (White 1980).

While whole-body hyperthermia was effective, its application as a medical therapy would be cumbersome and risky. Our lab therefore had been seeking a clinically relevant agent to induce this protective response. Many candidate chemicals and drugs were known to induce stress protein production in tissue culture cells but were too toxic for human use, such as heavy metals and 2,4-DNP. We selected stannous chloride as a potential clinical therapy as it was a potent inducer of endothelial heme oxygenase, also known as HSP32, and resulted in protection against reactive oxygen species (Kappas and Maines 1976). Furthermore, stannous chloride was already being administered to humans as a component of technetium pyrophosphate radionuclide imaging.

In collaboration with S. House and P. Guidon, Jr. in 2001, we had the opportunity to test our hypothesis that acquired cytoprotection is an anti-inflammatory state and that heat shock proteins play a role in this response to inflammatory stress (House et al. 2001). We used intraperitoneal injections of stannous chloride into Wistar rats in a stress conditioning regimen to trigger vascular endothelium to acquire cytoprotection. Intravital microscopy was used to monitor hemodynamic parameters and to visualize microcirculation of leukocytes. Formyl-methionyl-leucyl-phenylalanine (FMLP) was added to exposed mesentery tissue to stimulate the initial stages of inflammation and mimic sepsis. During severe reactions, leukocytes adhere firmly to the venular endothelium and they migrate across the endothelial cell layer into traumatized tissue, as part of the process of leukocyte extravasation. One group of rats received heat shock, as a positive control to stimulate cytoprotection. The presence of HSP70 in aortic tissue was used as a biomarker for the state of acquired cytoprotection (thermotolerance). Gallium nitrate which has immunosuppressive activity but which does not induce heat shock proteins nor acquisition of cytoprotection was used as a negative control in a stress conditioning regimen. During FMLP suffusion, leukocyte-endothelial adhesion increased in placebo- and gallium nitrate-treated rats but not in heat shocked and stannous chloride-treated animals, consistent with an anti-inflammatory effect in the latter two groups. The inability of leukocytes to adhere firmly to the endothelium effectively closed the gate on potentially inflammatory cells entering a tissue. Whereas HSP70 was not detected in aortic tissue of placebo- and gallium nitrate-treated rats, HSP70 was detected in aortic tissues from heat shocked and stannous chloridetreated animals. We concluded that stannous chloride was capable of inducing both a cytoprotected state and an antiinflammatory state in laboratory rats and may be an ideal pharmaceutical for future stress conditioning studies.

We also recognized that systemic exposure to a very high level of oxygen (hyperbaric oxygen) was capable of inducing a cytoprotective response in tissues (Perdrizet 2016). This response involved the stimulation of the heat shock genes by transcription factor Hsf-1 and antioxidant defenses within human microvascular endothelial cells primarily by signaling by reactive oxygen species that activate the transcription factor Nrf-2 (Godman et al. 2010). Hyperbaric oxygen therapy (HBOT) has several systemic effects which may be effective in the treatment of COVID-19 disease. HBOT can directly reverse tissue hypoxia and is anti-inflammatory - both global pathologic features of COVID-19 disease. Most recently, HBOT reduced hepatic cytokine production in a rodent model of severe, polymicrobial sepsis (Halbach et al. 2019). HBOT has been shown to reduce circulating cytokine levels in individuals with Crohn's disease (Weisz et al. 1997). This reduction in inflammation is associated with a reduction in tissue edema in humans (Hammarlund et al. 1991). Furthermore, HBOT can directly reverse tissue level hypoxia that may not be treated by conventional methods of oxygen administration, including mechanical ventilation (Abbot et al. 1994). 
We are aware of several small anecdotal reports of the safe application of HBOT to benefit SARS-CoV-2-infected patients with manifestations of pulmonary injury and dysfunction. A first case report was published by Zhong Xiaoling and colleagues in Wuhan, China, and has been translated into English in an online version (https://drive.google.com/file/d/ 1IJoyao8uFCCQjOxGFC9yqWN6oL-YjoqX/view). The same group has also published a summary of a five-case study in Wuhan (https://drive.google.com/file/d/ 1pjtuT44daBvc8LubVYcR064PLpgIjiFY/view).

Currently several clinical trials have been initiated to determine the efficacy of HBOT in the treatment of COVD-19 disease. Preliminary results are expected by July/August 2020. No studies have tested stannous chloride as a cytoprotective agent of vascular endothelium in humans for any indication, including COVID-19 disease.

\section{References}

Abbot NC, Beck JS, Carnochan FM, Gibbs JH, Harrison DK, James PB, Lowe JG (1994) Effect of hyperoxia at 1 and 2 ATA on hypoxia and hypercapnia in human skin during experimental inflammation. J Appl Physiol 77(2):767-773

Collins PL, Hightower LE (1982) Newcastle disease virus stimulates the cellular accumulation of stress (heat shock) mRNAs and proteins. J Virol 44(2):703-707

Currie RW, White FP (1981) Trauma-induced protein in rat tissues: a physiological role for a "heat shock" protein? Science 214(4516): $72-73$

Fu Y, Cheng Y, Wu Y (2020) Understanding SARS-CoV-2-mediated inflammatory responses: from mechanisms to potential therapeutic tools. Virol Sin. https://doi.org/10.1007/s12250-020-00207-4

Godman CA et al (2010) Hyperbaric oxygen induces a cytoprotective and angiogenic response in human microvascular endothelial cells. Cell Stress Chaperones 15(4):431-442

Gopalakrishnan A, Mossaid A, Lo KB, Vasudevan V, McCullough PA, Rangaswami J (2020) Fulminant acute kidney injury in a young patient with novel coronavirus 2019. Cardiorenal Med. https://doi. org/10.1159/000508179

Grasselli G et al (2020) Baseline characteristics and outcomes of 1591 patients infected with SARS-CoV-2 admitted to ICUs of the Lombardy Region, Italy. JAMA 323(16):1574-1581

Guan W-j et al (2020) Clinical characteristics of coronavirus disease 2019 in China. N Engl J Med 382(18):1708-1720

Halbach JL et al (2019) Early hyperbaric oxygen therapy improves survival in a model of severe sepsis. Am J Phys Regul Integr Comp Phys 317(1):R160-R168

Hammarlund C et al (1991) Hyperbaric oxygen treatment of healthy volunteers with u.v.-irradiated blister wounds. Burns 17(4):296-301

Han S-B, Lee S-Y (2018) The link between Kawasaki disease shock syndrome and macrophage activation syndrome in terms of organ dysfunction observed in children with systemic inflammatory response syndrome. Paediatr Int Child Health 38(4):308-309

Hooper PL, Hightower LE, Hooper PL (2012) Loss of stress response as a consequence of viral infection: implications for disease and therapy. Cell Stress Chaperones 17(6):647-655
House SD et al (2001) Effects of heat shock, stannous chloride, and gallium nitrate on the rat inflammatory response. Cell Stress Chaperones 6(2):164-171

Huang C et al (2020) Clinical features of patients infected with 2019 novel coronavirus in Wuhan, China. Lancet 395(10223):497-506

Kappas A, Maines MD (1976) Tin: a potent inducer of heme oxygenase in kidney. Science 192(4234):60

Li H et al (2020) SARS-CoV-2 and viral sepsis: observations and hypotheses. Lancet 395(10235):1517-1520

Magro C et al (2020) Complement associated microvascular injury and thrombosis in the pathogenesis of severe COVID-19 infection: a report of five cases. Transl Res. https://doi.org/10.1016/j.trsl.2020. 04.007

Marrani E, Burns JC, Cimaz R (2018) How should we classify Kawasaki disease? Front Immunol 9. https://doi.org/10.3389/fimmu.2018. 02974

McGonagle D, O’Donnell JS, Sharif K, Emery P, Bridgewood C Immune mechanisms of pulmonary intravascular coagulopathy in COVID19 pneumonia. Lancet Rheumatol. https://doi.org/10.1016/S26659913(20)30121-1

Murthy S et al (2020) Care for critically ill patients with COVID-19. JAMA 323(15):1499-1500

Nathan $\mathrm{N}$ et al (2020) Atypical presentation of COVID-19 in young infants. Lancet 395(10235):1481

Perdrizet GA (1996) The heat shock response and organ transplantation. Transplant Rev 10(2):78-98

Perdrizet GA (2016) Preoperative stress conditioning in humans: is oxygen the drug of choice? Oxygen Transport to Tissue XXXVII. NY, Springer New York, New York

Perdrizet GA, Heffron TG, Buckingham FC, Salciunas PJ, Gaber AO, Stuart FP, Thistlethwaite JR (1989) Stress conditioning: a novel approach to organ preservation. Curr Surg 46:23-26

Ritossa F (1962) A new puffing pattern induced by temperature shock and DNP in drosophila. Experientia 18(12):571-573

Sciascia S et al (2020) Pilot prospective open, single-arm multicentre study on off-label use of tocilizumab in severe patients with COVID-19. Clin Exp Rheumatol 38:00-00

Solomon JM, Rossi JM, Golic K, McGarry T, Lindquist S (1991) Changes in hsp70 alter thermotolerance and heat-shock regulation in Drosophila. New Biologist 3(11):1106-1120

Varga Z, Flammer AJ, Steiger P, Haberecker M, Andermatt R, Zinkernagel AS, Mehra MR, Schuepbach RA, Ruschitzka F, Moch H (2020) Endothelial cell infection and endotheliitis in COVID-19. Lancet 395(10234):1417-1418

Weisz G, Lavy A, Adir Y, Melamed Y, Rubin D, Eidelman S, Pollack S (1997) Modification of in vivo and in vitro TNF- $\alpha$, IL-1, and IL-6 secretion by circulating monocytes during hyperbaric oxygen treatment in patients with perianal Crohn's disease. J Clin Immunol 17(2):154-159

White FP (1980) Differences in protein synthesized in vivo and in vitro by cells associated with the cerebral microvasculature. A protein synthesized in response to trauma? Neuroscience 5(10):1793-1799

White FP (1981) The induction of stress proteins in organ slices from brain, heart, and lung as a function of postnatal development. J Neurosci 1(11):1312

Publisher's note Springer Nature remains neutral with regard to jurisdictional claims in published maps and institutional affiliations. 